\title{
Clarifying the Relationship between Psychopathy and Intelligence using Four Dimensions of the WASI-II
}

\author{
Kathryn Sharratt ${ }^{1}$, Daniel Boduszek ${ }^{1,2} \&$ Christopher Retzler $^{1}$ \\ ${ }^{1}$ University of Huddersfield, Huddersfield, United Kingdom \\ ${ }^{2}$ SWPS University of Social Sciences and Humanities, Katowice, Poland
}

Paper accepted for publication in Deviant Behavior

\section{Correspondence to:}

Kathryn Sharratt

Address: University of Huddersfield, Queensgate, Huddersfield, HD1 4DH, United Kingdom Tel: +44 (0) 1484472886

Email: k.sharratt@hud.ac.uk 


\begin{abstract}
Empirical evidence concerning the relationship between psychopathy and intelligence remains equivocal. The aim of the current study was to clarify the nature of the relationship by examining the association between four facets of the Psychopathic Personality Traits Scale (PPTS) and the Wechsler Abbreviated Scale of Intelligence - Second Edition (WASI-II). Based on a non-institutionalised sample $(\mathrm{N}=253)$, Affective Responsiveness demonstrated a significant negative association with Similarities, whereas Interpersonal Manipulation demonstrated a significant positive association with Vocabulary. Egocentricity demonstrated a significant negative relationship with both Vocabulary and Matrix Reasoning. Implications of these findings for the assessment of psychopathy and intelligence are discussed.
\end{abstract}

Keywords: Psychopathy; intelligence; WASI-II; Psychopathic Personality Traits Scale; confirmatory factor analysis 


\section{Introduction}

Fictional representations of psychopathy have captured the attention of audiences for decades. These characters are often depicted as more intelligent than the average citizen, and Anthony Hopkins' portrayal of Hannibal Lecter in Silence of the Lambs is a particularly memorable example. Lecter is a serial killer with cannibalistic tendencies who demonstrates an extraordinary ability to outsmart the Federal Bureau of Investigation (FBI). Many of the traits that are bestowed to Lecter also feature in academic accounts of psychopathy, including a lack of empathy and remorse, superficial charm, a sense of grandiose and pathological egocentricity (Cleckey 1941). Consistent with media representations, Cleckley also believed that psychopathy is associated with superior levels of intelligence, and this was hypothesised to facilitate the ability to manipulate and deceive others in the pursuit of personal gains.

In contrast, empirical research has revealed weak correlations between overall scores on the Psychopathy Checklist - Revised (PCL-R; Hare 2003) and measures of intelligence (see Hare 2003 for a review), thus failing to confirm the popular belief that psychopathic individuals demonstrate above average intelligence. The PCL-R relies heavily on the assessment of behavioural indicators of psychopathy, including erratic lifestyles and criminal and antisocial tendencies. However, it is important to remember that whilst the contravention of legal and social norms might be a potential outcome of psychopathy for some individuals, there is also a growing body of evidence demonstrating that psychopathic traits are present in non-criminal samples such as corporate professionals, law enforcement officers and presidential candidates (Debowska, Boduszek, Dhingra, Willmott, Sherretts and DeLisi 2018; Lilienfeld, Waldman, Landfield, Rubenzer and Faschingbauer 2012; Body, Ladyshewsky and Galvin 2010).

Bearing in mind that psychopathy can be identified in both criminal and non-criminal samples, some scholars have suggested that the core essence of the phenomenon might be better captured by affective and interpersonal characteristics (e.g. shallow affect and the inability to establish a genuine emotional connection with others) since these can be universally applied to 
both groups (Boduszek and Debowska, 2016). However, when the combined affectiveinterpersonal factor of the PCL-R was examined among psychiatric patients, those who scored highly actually displayed lower levels of intelligence (O'Kane, Fawcett and Blackburn 1996), thus directly contradicting common beliefs that psychopathy is associated with elevated intelligence. Meanwhile, in a sample of university students, there was no direct relationship between the affective-interpersonal dimension and intelligence (Bate, Boduszek, Dinghra and Bale 2014).

More recent empirical research has demonstrated that the affective and interpersonal characteristics constitute distinct facets of psychopathy (Boduszek and Debowska 2016; Debowska, Boduszek and Woodfield 2019), emphasising the importance of examining these factors independently of one another. Indeed, analysis based on offenders and psychiatric patients has shown that the interpersonal items are positively related to intelligence, whereas the affective items are negatively related to intelligence (DeLisi, Vaughn, Beaver and Wright 2010; Salekin, Neumann, Leistico and Zalot 2004; Vitacco, Neumann and Jackson 2005; Vitacco, Neumann and Wodushek 2008). Therefore, it would appear that the connection between psychopathy and intelligence might be less straightforward than first thought, and whilst interpersonal characteristics are associated with elevated intelligence, the opposite is true for affective traits. However, in a community sample, Neumann and Hare (2008) revealed negative correlations with both affective and interpersonal dimensions, allowing for the possibility that the pattern of relationships might vary according to sample characteristics.

The desire for a method of assessment that alleviates items relating to criminal/antisocial deviance prompted the development of the Psychopathic Personality Traits Scale (PPTS; Boduszek, Debowska, Dhingra and DeLisi 2016). The PPTS retains subscales relating to Affective Responsiveness (AR) and Interpersonal Manipulation (IPM), but it also includes other important characteristics of psychopathy that were missing from the PCL-R, namely Cognitive Responsiveness (CR) and Egocentricity (EGO). CR measures the inability 
to understand the emotional state of others, mentally represent another person's emotional processes, and emotionally engage with others at a cognitive level. EGO, on the other hand, relates to the tendency to focus on one's own interests, beliefs and attitudes. The PPTS demonstrates good construct validity as indicated by overall model fit statistics $(\mathrm{CFI}=0.96$; $\mathrm{TLI}=0.95 ; \mathrm{RMSEA}=0.04)$ and statistically significant item loadings produced during confirmatory factor analysis (Boduszek et al. 2016). The factor structure was further confirmed through a pattern of differential relationships with external variables, however, the relationships between the four factors of the PPTS and intelligence is currently unknown.

Although increasing attention has been paid to the dimensionality of psychopathy, there is limited evidence regarding the relationship with different types of intelligence. One of the aforementioned studies relied on full-scale intelligence scores (Vitacco et al. 2008), whilst others were restricted to the analysis of data pertaining to verbal intelligence only (DeLisi et al. 2010; Neumann and Hare 2008; Vitacco et al. 2005). Therefore, it is unclear whether psychopathy is associated with advantages in all domains of intelligence, or just in relation to certain abilities.

The Wechsler Abbreviated Scale of Intelligence - Second Edition (WASI-II; Wechsler 2011) can be administered in just 15-30 minutes and yields scores for both Perceptual Reasoning (PR) and Verbal Comprehension (VC), therefore representing a convenient method of assessment that retains some degree of multidimensionality. PR relates to the ability to analyse and synthesise abstract visual stimuli, whereas VC measures word knowledge and verbal concept formation. Confirmatory factor analysis (CFA) of the original standardisation sample revealed a two-factor model comprising of PR and VC fit the data better than a unidimensional solution, however, dissatisfaction arises with the Manual since there is no evidence that alternative model conceptualisations were tested (see for example, Gignac and Watkins 2013). Furthermore, the factor structure of the WASI-II has not been independently examined to ascertain whether the preferred model can be replicated. Exploratory factor 
analysis (EFA) of the first edition of the WASI (Wechsler 1999) supported the distinction between PR and Verbal VC factors among a group of patients referred for neuropsychological assessment (Ryan, Carruthers, Miller, Souheaver, Gontkovsky and Zehr 2003), but there is no guarantee that these observations can be generalised to the more recent edition of the scale or non-clinical samples. Although analyses of the first edition WASI are available in non-clinical samples, one reported a spuriously low chi square value relative to other model fit statistics (Abu-Hilal, Al-Baili, Sartawi, Abdel-Fattah and Al-Qaryouti 2011), and the other performed a joint analysis with a second intelligence test (Canivez, Konold, Collins and Wilson 2009) that would only be applicable in circumstances where the two batteries are administered together.

Therefore, the aim of the present study was to examine the relationship between the four facets of psychopathy measured by the PPTS and the WASI-II. The current analysis was based on a university sample and therefore adds valuable evidence regarding the relationship between psychopathy and intelligence in non-institutionalised samples. Given that the factor structure of the WASI-II has not been independently confirmed, an additional aim was to compare a series of model conceptualisations, including previously untested solutions. No prior assumptions were made about the nature of the relationship between the PPTS and WASI-II since the factor structure of the latter was yet to be established.

\section{Method}

\section{Participants}

The opportunistic sample consisted of 253 participants, including university students $(\mathrm{N}=210 ; 83.00 \%)$ and $\operatorname{staff}(\mathrm{N}=26 ; 10.28 \%)$, and acquaintances of the researcher $(\mathrm{N}=17$; $6.72 \%)$. Participants ranged in age from 16 to $82(\mathrm{M}=28.26, \mathrm{SD}=13.40)$. The sample consisted of 183 females and 70 males. The study received approval from the institution's ethics panel and all participants were required to provide written consent. 


\section{Materials}

The Psychopathic Personality Traits Scale (PPTS; Boduszek et al. 2016) is a self-report scale requiring participants to agree (0) or disagree (1) with 20 items concerning their knowledge, skills, attitudes and beliefs. Items load onto four subscales (Affective Responsiveness; AR; Cognitive Responsiveness; CR; Interpersonal Manipulation; IPM; Egocentricity; EGO) and higher scores indicate elevated levels of interpersonal manipulation and egocentricity, and greater deficits in affective and cognitive responsiveness. Composite reliability estimates indicate good internal reliability for the four subscales $(\mathrm{AR}=0.86 ; \mathrm{CR}=$ $0.76 ; \mathrm{IPM}=0.84 ; \mathrm{E}=0.69)$.

The Wechsler Abbreviated Scale of Intelligence - Second Edition (WASI-II; Wechsler 2011 ) is suitable for use with 6 - 90 year olds and is recommended for use in research settings where only a brief estimate of intellectual functioning is required. The WASI-II contains four subtests known as Block Design (MR), Vocabulary (V), Matrix Reasoning (MR) and Similarities (S). The BD and MR subtests combine to produce the Perceptual Reasoning Index (PRI), and the V and S subtests belong to the Verbal Comprehension Index (VCI). The four subtests were administered and scored by a psychologist in accordance with the instructions presented in the Manual (Wechsler 2011). The subtests are scaled in T score units $(M=50, S D$ $=10$ ) whereas the Full Scale Intelligence Quotient - Four Subtest (FSIQ4) is scaled in traditional IQ units $(\mathrm{M}=100, \mathrm{SD}=15)$.

\section{Analysis}

The dimensionality of the WASI-II was investigated using confirmatory factor analysis (CFA) and bifactor modelling techniques with robust maximum likelihood (MLR) estimation in Mplus version 7.11 (Muthen and Muthen 1998-2017). The first-order models were (1) a unidimensional model; (2) a two-factor model comprising PRI and VCI; (3) a three-factor model with PRI and separate factors reflecting the V and S subtests; (4) a three-factor model with VCI and separate factors reflecting the BD and MR subtests; and (5) a four-factor model 
reflecting the original $\mathrm{BD}, \mathrm{MR}, \mathrm{V}$ and $\mathrm{S}$ subtests. All of the bifactor models has just one general factor but variously included (6) two grouping factors reflecting PRI and VCI; (7) three grouping factors reflecting PRI and the V and S subtests; (8) three grouping factors reflecting VCI and the BD and MS subtests; and (9) four grouping factors reflecting the BD, MR, V and S subtests.

The following items were excluded from the $\operatorname{BD}(1,2), \operatorname{MR}(1,2,3), \mathrm{V}(1,2,3,31)$ and $\mathrm{S}(1,2,3)$ subtests due to a lack of variance. The remaining 88 items were computed into 29 radial parcels by randomly selecting items from the same hypothesised factor. As can be seen in Table 1, parcels contained three items up until items loading onto that factor were exhausted, in which case final parcels contained two items.

Overall model fit was assessed using a range of goodness-of-fit statistics and the appropriateness of the model parameters. The chi-square $\left(\chi^{2}\right)$ statistic assessed the sample and implied covariance matrix; a good fitting model is indicated by a non-significant result. The chi-square statistic is, however, strongly associated with sample size, and as such good models tend to be over-rejected. The Comparative Fit Index (CFI; Bentler 1990) and the Tucker Lewis Index (TLI; Tucker and Lewis 1973) are measures of how much better the model fits the data compared to a baseline model where all variables are uncorrelated. For these indices values above 0.95 indicate good model fit but values above 0.9 are considered acceptable (Bentler 1990; Hu and Bentler 1999). The standardized root mean-square residual (SRMR; Joreskog and Sorborn 1981) and the root mean-square error of approximation (RMSEA; Steiger 1990) are also presented. Ideally these indices should be less than 0.05 , but values less than 0.08 are considered acceptable (Bentler 1990; Hu and Bentler 1999). 
RELATIONSHIP BETWEEN PSYCHOPATHY AND INTELLIGENCE

Table 1

WASI-II Items assigned to parcels

\begin{tabular}{|c|c|c|c|c|c|c|c|c|c|}
\hline & Parcel 1 & Parcel 2 & Parcel 3 & Parcel 4 & Parcel 5 & Parcel 6 & Parcel 7 & Parcel 8 & Parcel 9 \\
\hline $\mathrm{BD}$ & $7,12,13$ & $3,5,6$ & $4,8,10$ & 9,11 & - & - & - & - & - \\
\hline MR & $4,24,25$ & $14,18,26$ & $12,16,19$ & $11,13,17$ & $7,21,22$ & $23,29,30$ & $8,9,15$ & $20,27,28$ & $5,6,10$ \\
\hline V & $14,19,28$ & $6,9,11$ & $13,14,27$ & $12,23,25$ & $7,16,17$ & $20,21,29$ & $10,18,24$ & $22,26,30$ & $5,8,15$ \\
\hline S & $5,9,23$ & $13,15,21$ & $4,11,14$ & $16,20,24$ & $6,12,19$ & $7,10,18$ & $8,17,22$ & - & - \\
\hline
\end{tabular}

Note $: \mathrm{BD}=$ Block Design; $\mathrm{MR}=$ Matrix Reasoning; $\mathrm{V}=$ Vocabulary; $\mathrm{S}=$ Similarities . 


\section{Results}

Descriptive statistics for the PPTS and WASI-II are presented in Table 2. Participants scored low on all four factors of psychopathy and intelligence scores were close to the average for the general population.

Table 2

Descriptive statistics for the PPTS and WASI-II

\begin{tabular}{|c|c|c|c|c|c|}
\hline & $M$ & $S D$ & $M d n$ & Min & Max \\
\hline AR & 1.01 & 1.25 & 1.00 & 0 & 5 \\
\hline $\mathrm{CR}$ & 0.79 & 1.07 & 0.00 & 0 & 5 \\
\hline IPM & 2.36 & 1.59 & 2.00 & 0 & 5 \\
\hline EGO & 1.89 & 1.14 & 2.00 & 0 & 5 \\
\hline $\mathrm{BD}$ & 50.14 & 9.85 & 50.00 & 23 & 80 \\
\hline MR & 48.35 & 7.27 & 49.00 & 21 & 80 \\
\hline V & 53.20 & 10.07 & 53.00 & 26 & 80 \\
\hline S & 47.30 & 7.27 & 47.00 & 30 & 80 \\
\hline FSIQ4 & 99.41 & 11.69 & 98.00 & 71 & 160 \\
\hline
\end{tabular}

Note: $\mathrm{AR}=$ Affective Responsiveness; $\mathrm{CR}=$ Cognitive Responsiveness; IPM $=$ Interpersonal Manipulation; EGO = Egocentricity; $\mathrm{BD}=$ Block Design; $\mathrm{MR}=$ Matrix Reasoning; V = Vocabulary; $\mathrm{S}=$ Similarities; FSIQ = Full Scale Intelligence Quotient - Four Subtest. 


\section{Factor Structure and Composite Reliability of the WASI-II}

Table 3 reports the fit indices for the nine alternative models of the WASI-II. None of the models provided a good approximation of the data based on CFI and TLI statistics, but models 4, 7, 8 and 9 approached the criteria for an adequate fitting model. Further inspection of the RMSEA and SRMR revealed model 8 to be superior, and these statistics were acceptable and approached the criteria for good model fit. Thus, a bifactor model with four grouping factors reflecting the original subtests was considered to be the best fitting model overall.

The adequacy of this model can also be determined in relation to its parameter estimates. All parcels displayed statistically significant $(p<0.01)$ average standardised factor loadings. All factor loadings were in the expected direction, but only the grouping factors exceeded $0.4(\mathrm{FSIQ}=0.15 ; \mathrm{BD}=0.76 ; \mathrm{MR}=\mathrm{M}=0.49 ; \mathrm{V}=0.58 ; \mathrm{S}=0.50)$. According to Reise, Moore and Haviland (2010), when items load more strongly onto the grouping factors than the general factor, the grouping factors should be regarded as the primary level of interpretation.

The grouping factors were found to be associated with one another in the expected manner. BD was most strongly correlated with MR $(r=0.50, p<0.001)$, whereas V was most strongly correlated with $\mathrm{S}(\mathrm{r}=0.54, \mathrm{p}<0.001)$. Moderate correlations were also observed between $\mathrm{BD}$ and $\mathrm{S}(\mathrm{r}=0.32, \mathrm{p}<0.001)$ and $\mathrm{MR}$ and $\mathrm{S}(\mathrm{r}=0.32, \mathrm{p}<0.001)$. However, none of the correlations were so high to indicate that they measure the same phenomenon. Relationships between BD and V $(r=0.20, p<0.001)$ and MR and V $(r=0.23, p<0.001)$ were weaker in comparison.

Traditional measures such as Cronbach's alpha have been criticised within a latent variable modelling context given the propensity to over- or under-estimate scale reliability (see Raykov, 1998). In order to provide a more rigorous assessment of internal reliability, the current study calculated composite reliability using the following formula where $C R=$ 
reliability of the factor score, $\lambda_{\mathrm{i}}=$ standardized factor loading, and $\theta_{\mathrm{i}}=$ standardised error variance:

$$
\mathrm{CR}=\frac{\left(\sum \lambda_{i}\right)^{2}}{\left(\sum \lambda_{i}\right)^{2}+\sum \operatorname{Var}\left(\varepsilon_{i}\right)}
$$

Values greater than 0.60 are generally considered acceptable (Bagozzi and Yi 1988; Diamantopoulos and Siguaw 2000). Composite reliability was low for the general factor $(0.41)$ but high for the BD (0.85), MR (0.75), V (0.83) and S (0.70) grouping factors.

\section{Relationship between Psychopathy and Intelligence}

Path analysis was performed to examine the relationship between the four facets of psychopathy as measured by the PPTS and the four WASI-II grouping factors (Figure 1). V demonstrated a significant association with IPM $(\beta=0.12, p<0.05)$ and $E(\beta=-0.13, p<$ 0.05), albeit in opposite directions. MR demonstrated a significant negative association with $\mathrm{E}$ $(\beta=-0.07, \mathrm{p}<0.05)$, whereas $\mathrm{S}$ demonstrated a significant negative association with $\mathrm{AR}(\beta=$ $-0.11, \mathrm{p}<0.05)$. BD did not possess significant associations with any of the psychopathy factors. 
Table 3

Fit Indices for Five Alternative Models of the WASI-II

\begin{tabular}{|c|c|c|c|c|c|c|}
\hline & $\chi^{2}$ & $d f$ & CFI & TLI & $\begin{array}{l}\text { RMSEA (90\% } \\
\text { CI) }\end{array}$ & SRMR \\
\hline 1. Unidimensional Model & $1573.70 * * *$ & 377 & 0.38 & 0.33 & $\begin{array}{c}0.112 \\
(0.106 / 0.118)\end{array}$ & 0.130 \\
\hline 2. Two Factor Model & $1481.90 * * *$ & 376 & 0.43 & 0.38 & $\begin{array}{c}0.108 \\
(0.102 / 0.114)\end{array}$ & 0.142 \\
\hline 3. Three Factor Model $(P R I+V+S)$ & $808.61 * * *$ & 374 & 0.78 & 0.76 & $\begin{array}{c}0.068 \\
(0.061 / 0.074)\end{array}$ & 0.072 \\
\hline 4. Three Factor Model (VCI + BD + MR) & $724.80 * * *$ & 374 & 0.82 & 0.80 & $\begin{array}{c}0.061 \\
(0.054 / 0.067)\end{array}$ & 0.069 \\
\hline 5. Four Factor Model & $642.30 * * *$ & 371 & 0.86 & 0.85 & $\begin{array}{c}0.054 \\
(0.047 / 0.061)\end{array}$ & 0.061 \\
\hline 6. Bifactor Model (2 grouping factors) & $697.09 * * *$ & 347 & 0.82 & 0.79 & $\begin{array}{c}0.063 \\
(0.056 / 0.070)\end{array}$ & 0.060 \\
\hline 8. Bifactor Model (3 grouping factors PRI $+\mathrm{V}+\mathrm{S}$ ) & $612.01 * * *$ & 345 & 0.86 & 0.84 & $\begin{array}{c}0.055 \\
(0.048 / 0.062)\end{array}$ & 0.057 \\
\hline $\begin{array}{l}\text { 7. Bifactor Model ( } 3 \text { grouping factors VCI + BD + } \\
\text { MR) }\end{array}$ & $617.94 * * *$ & 345 & 0.86 & 0.83 & $\begin{array}{c}0.056 \\
(0.049 / 0.063)\end{array}$ & 0.055 \\
\hline 9. Bifactor Model (4 grouping factors) & $586.25 * * *$ & 342 & 0.87 & 0.85 & $\begin{array}{c}0.053 \\
(0.046 / 0.060)\end{array}$ & 0.053 \\
\hline
\end{tabular}

$\chi^{2}=$ chi square goodness of fit statistic; $d f=$ degrees of freedom; CFI = Comparative Fit Index; TLI = Tucker Lewis Index; RMSEA $=$ RootMean-Square Error of Approximation; $\mathrm{CI}=$ Confidence Interval; SRMR = Standardized Root Mean Square Residual.

$* * *$ indicates $\chi^{2}$ is statistically significant $(p<.001)$. 


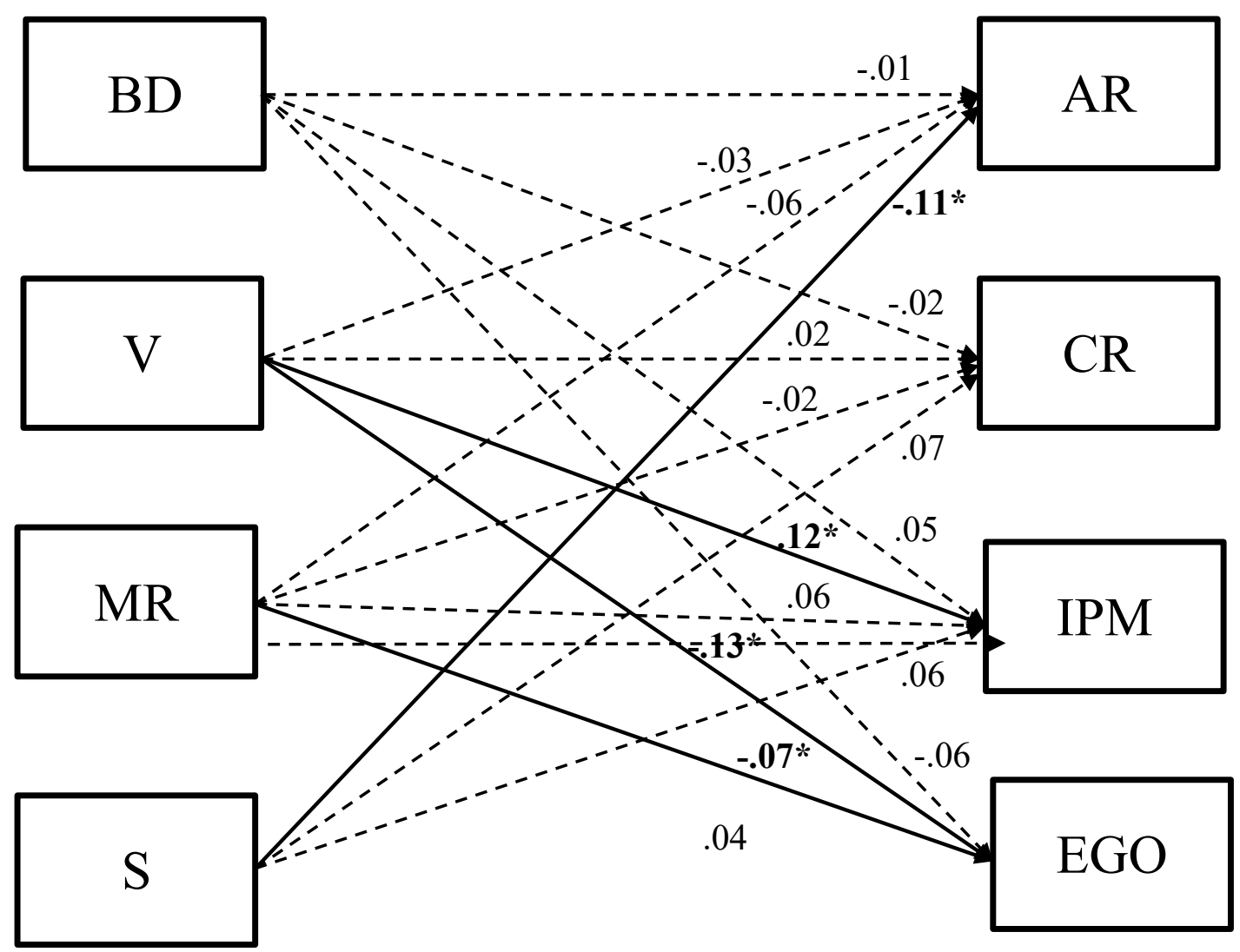

Figure 1. Relationship between four factors of the PPTS and WASI-II. AR = Affective Responsiveness; $\mathrm{CR}=$ Cognitive Responsiveness; IPM = Interpersonal Manipulation; EGO = Egocentricity; $\mathrm{BD}=$ Block Design; $\mathrm{MR}=$ Matrix Reasoning; $\mathrm{V}=$ Vocabulary; $\mathrm{S}=$ Similarities. $* \mathrm{p}<0.05$

\section{Discussion}

The aim of the present study was to examine the nature of the relationship between psychopathy and intelligence, as measured by the PPTS and WASI-II. Given that the factor structure of the WASI-II had not been independently confirmed, a precursor was to compare a series of model conceptualisations using traditional CFA and bifactor techniques. On the basis of a range of goodness-of-fit statistics, a bifactor model with a single general factor and four grouping factors reflecting the original subtests was found to provide the best fit for the data. Average standardised factor loadings were significantly higher for the grouping factors than the general factor, indicating that the former should be regarded as the primary level of interpretation (Reise et al. 2010). This observation is broadly consistent with previous studies 
demonstrating that the Wechsler Adult Intelligence Scale - Fourth Edition (WAIS-IV; Wechsler 2008) is also best represented by a bifactor model, with the exception that subtests loaded more strongly onto the general factor for the full-scale version (Gignac and Watkins 2013).

The various facets of psychopathy did not possess any significant relationships with Block Design, a finding that coincides with other studies demonstrating the absence of a relationship between psychopathy and non-verbal performance (Salekin et al. 2004). Affective Responsiveness, however, demonstrated a significant negative association with Similarities. This is consistent with previous studies that have also revealed an inverse relationship between affective traits of psychopathy and verbal abilities (e.g. DeLisi et al. 2010; Salekin et al. 2004; Vitacco, et al. 2008). This observation can be interpreted in light of evidence demonstrating a positive association between verbal intelligence and empathy (Joliffe and Farrington 2006). Joliffe and Farrington reasoned that enhanced verbal skills facilitate effective communication and the ability to comprehend how other people are feeling. Thus, the negative relationship between Affective Responsiveness and verbal abilities might partly explain why individuals scoring highly on this component of psychopathy experience a reduced ability to internalise affective states.

In contrast, Interpersonal Manipulation demonstrated a significant positive association with Vocabulary. Again, this is consistent with previous research that has revealed a positive relationship between the interpersonal component of psychopathy and verbal skills (e.g. DeLisi et al. 2010; Salekin et al. 2004; Vitacco, et al. 2008). Research in the field of deception has demonstrated that verbal fluency is positively related to ratings of truthfulness (DePaulo, Lindsay, Malone, Charlton and Cooper 2003). Therefore, it seems likely that enhanced verbal skills facilitate the ability to convincingly deceive, manipulate and charm others, which are characteristic of the Interpersonal Manipulation component of psychopathy. In relation to this, Boduszek, Debowska and Willmott (2017) found that offenders who scored highly on 
Interpersonal Manipulation subscale of the PPTS were more likely to be imprisoned for whitecollar crimes than other offence categories. However, Boduszek and colleagues did not control for intelligence and this raises the question whether these offenders actually possessed lower levels of verbal intelligence, and hence were less successful in their ability to deceive others in the pursuit of financial gains.

Meanwhile, Egocentricity was negatively correlated with both Vocabulary and Matrix Reasoning. As noted previously, verbal skills are positively associated with the ability to comprehend others' perspectives and experiences (Joliffe and Farrington 2006). Conversely, if impaired verbal skills decrease the ability to understand others' perspectives, this might increase the likelihood that an individual focuses on their own interests and beliefs (as is the case in egocentricity). Egocentricity is also associated with cognitive rigidity - the inability to switch between different perspectives or the tendency to use a limited range of strategies when deriving a solution to a problem (Curry, Chesters and Viding, 2011). This might explain why individuals who scored more highly on Egocentricity experienced greater difficulties with Matrix Reasoning since this subtest requires the deployment of inductive or fluid reasoning to solve novel abstract puzzles. A noteworthy study by Schweinle, Ickes, Rollings and Jacquot (2010) revealed that men who were violent towards their partners displayed increased egocentric traits. Interpreted in light of the present findings, it is possible that associated impairments in cognitive reasoning meant that these men were less able to identify non-violent solutions to perceived problems within their relationship.

The observation of a distinct pattern of relationships between psychopathic traits and intellectual abilities emphasises the importance of controlling for intelligence in the assessment of psychopathy (see Boduszek, Debowska and Willmott 2019 for a discussion). This is further illustrated by an experimental study demonstrating that individuals who scored highly on both psychopathy and intelligence produced normative physiological responses to emotionally evocative stimuli (Bate et al. 2014). The above commentary also demonstrates how the 
inclusion of intelligence in empirical research has the potential to offer valuable insights into explanatory mechanisms for behavioural and interpersonal manifestations of psychopathy. However, it is important to note that intelligence can act as either a 'risk' or 'protective' factor in the relationship between psychopathy and external variables. For example, Johansson and Kerr (2005) discovered that male offenders who scored high on psychopathy and high on intelligence were more likely to begin offending at a young age, whereas Beggs and Grace (2008) found that offenders who scored high on psychopathy and high on intelligence less likely to be reconvicted for sexual offences.

Although this was the first study to independently examine the factor structure of the WASI-II and test the relationship with four facets of the PPTS, it is not without limitations. Firstly, the collection of cross-sectional data meant that the direction of the relationship between psychopathy and intelligence could not be established. Secondly, analysis was based on an opportunistic sample mostly comprising of university staff and students. Hence, participants typically scored low on psychopathy and obtained intelligence scores close to the mean. Future research should endeavor to include samples representative of the general population, and additional research is also required to ascertain whether the current pattern of relationships is generalizable to clinical and forensic settings with more diverse representations of psychopathy and intelligence.

In summary, the WASI-II is best represented by a bifactor model comprising of a single general factor and four grouping factors, albeit with significantly higher average standardised factor loadings for the grouping factors. The four grouping factors displayed a unique pattern of relationship with the four psychopathy factors. Affective Responsiveness demonstrated a significant negative association with Similarities, whereas Interpersonal Manipulation demonstrated a significant positive association with Vocabulary. Meanwhile, Egocentricity demonstrated a significant negative relationship with both Vocabulary and Matrix Reasoning. As discussed above, these findings have important implications for the assessment of 
psychopathy, and it is recommended that future studies endeavour to account for intelligence as this could provide valuable insights into explanatory mechanisms for behavioural and interpersonal manifestations of psychopathy.

\section{References}

Abu-Hilal, Maher M., Mohammad A. Al-Baili, AbdelAziz Sartawi, Faisal Abdel-Fattah, and Ibrahim A. Al-Qaryouti. 2011. "Psychometric Properties of the Wechsler Abbreviated Scale of Intelligence (WASI) with an Arab Sample of School Students.” Individual Differences Research 9(4): 219-230.

Bagozzi, Richard P., and Youjae Yi. 1988. "On the evaluation of structural equation models." Journal of the Academy of Marketing Science 16(1): 74-94

Bate, Carolyn, Daniel Boduszek, Katie Dhingra, and Christopher Bale. 2014. "Psychopathy, intelligence and emotional responding in a non-forensic sample: an experimental investigation." The Journal of Forensic Psychiatry and Psychology 25(5): 600-612.

Beggs, Sarah M., and Randolph C. Grace. 2008. "Psychopathy, intelligence, and recidivism in child molesters. Evidence of an interaction effect." Criminal Justice and Behavior 35: 683-695.

Bentler, Peter M. 1990. "Comparative fit indices in structural models.” Psychological Bulletin 107(2): 238-246

Boduszek, Daniel, Agata Debowska, and Dominic Willmott. 2019. "Psychopathic Personality Traits Model (PPTM): A new approach to defining psychopathy.” In M. DeLisi (Ed.), Routledge International Handbook of Psychopathy and Crime (pp. 216 - 224). Routledge Taylor and Francis Group

Boduszek, Daniel, Agata Debowska, and Dominic Willmott. 2017. "Latent profile analysis of psychopathic traits among homicide, general violent, property, and white-collar offenders." Journal of Criminal Justice 51: 17-23. 
RELATIONSHIP BETWEEN PSYCHOPATHY AND INTELLIGENCE

Boduszek, Daniel and Agata Debowska. 2016. "Critical evaluation of psychopathy measurement (PCL-R and SRP-III/SF) and recommendations for future research." Journal of

Criminal Justice 44: 1-12.

Boduszek, Daniel, Agata Debowska, Katie Dhingra, and Matt DeLisi. 2016. "Introduction and validation of Psychopathic Personality Traits Scale (PPTS) in a large prison sample." Journal of Criminal Justice 46: 9-17.

Boddy, Clive RP, Richard Ladyshewsky, and Peter Galvin. 2010. "Leaders without ethics in global business: Corporate psychopaths.” Journal of Public Affairs 10(3): 121-138.

Canivez, Gary L., Timothy R. Konold, Jason M. Collins, and Greg Wilson. 2009. "Construct Validity of the Wechsler Abbreviated Scale of Intelligence and Wide Range Intelligence Test: Convergent and Structural Validity.” School Psychology Quarterly 24(4): 252-265.

Cleckley, Harvey. 1941. The mask of sanity (1st ed.). St. Louis, MO: C.V. Mosby.

Curry, Oliver, Matthew Jones Chesters, and Essi Viding. 2011. "The psychopath's dilemma: The effects of psychopathic personality traits in one-shot games." Personality and Individual Differences 50: 804-809.

Debowska, Agata, Daniel Boduszek, Katie Dhingra, Nicole Sherretts, Dominic Willmott, and Matt DeLisi. 2018. "Can we use Hare’s psychopathy model within forensic and nonforensic populations? An empiricalinvestigation.” Deviant Behavior 39(2): 224-242.

Debowska, Adata, Daniel Boduszek and Russell Woodfield. 2019. The PCL-R family of psychopathy measures: Dimensionality and predictive utility of the PCL-R, PCL: SV, PCL: YV, SRP-III, and SRP-SF. In M. DeLisi (Ed.), Routledge International Handbook of Psychopathy and Crime (pp. 225 - 240). Routledge Taylor \& Francis Group

DeLisi, Matt, Michael G. Vaughn, Kevin M. Beaver, and John Paul Wright. 2010. "The Hannibal Lecter myth: Psychopathy and verbal intelligence in the MacArthur Violence 
RELATIONSHIP BETWEEN PSYCHOPATHY AND INTELLIGENCE

Risk Assessment Study." Journal of Psychopathology and Behavioral Assessment 32(2): 169-177.

DePaulo, B., Lindsay, J., Malone, B.E., Muhlenbruck, L., Charlton, K., and Cooper, H. 2003. “Cues to Deception.” Psychological Bulletin 129(1): 74-118.

Diamantopoulos, Adamantios, and Judy A. Siguaw. 2000. Introducing LISREL. London: Sage Publications.

Gignac, Gilles E., and Marley W. Watkins. 2013. "Bifactor Modeling and the Estimation of Model-Based Reliability in the WAIS-IV." Multivariate Behavioral Research 48(5): 639-662.

Hare, Robert. 2003. The Hare Psychopathy Checklist - Revised. Toronto: Multi- Health Systems.

Johansson, Peter, and Margaret Kerr. 2005. "Psychopathy and intelligence: a second look." Journal of Personality Disorders 19: 357-369.

Jolliffe, Darrick, and David P. Farrington. 2006. "Development and validation of the Basic Empathy Scale." Adolescence 29(4): 589-611.

Joreskog, Karl and Dag Sorbom. 1981. LISREL V: Analysis of linear structural relationships by the method of maximum likelihood. Chicago: National Educational Resources.

Lilienfeld, Scott O., Irwin D. Waldman, Kristin Landfield, Ashley L. Watts, Steven Rubenzer, and Thomas R. Faschingbauer. 2012. "Fearless dominance and the US presidency: Implications of psychopathic personality traits for successful and unsuccessful political leadership." Journal of Personality and Social Psychology 103(3): 489-505.

Neumann, Craig S., and Robert D. Hare. 2008. "Psychopathic traits in a large community sample: Links to violence, alcohol use, and intelligence." Journal of Consulting and Clinical Psychology 76: 893-899.

Muthén, Linda K., and Bengt O. Muthén. 1998. Mplus - Statistical analysis with latent variables. User's guide (6th ed.). Los Angeles: Muthen and Muthen 
RELATIONSHIP BETWEEN PSYCHOPATHY AND INTELLIGENCE

O'Kane, Aisling, Diane Fawcett, and Ronald Blackburn. 1996. "Psychopathy and moral reasoning: comparison of two classifications." Personality and Individual Differences 20: $515-514$.

Raykov, Tenko. 1997. "Estimation of composite reliability for congeneric measures.” Applied Psychological Measurement 21(2): 173-184.

Reise, Steven P., Tyler M. Moore, and Mark G. Haviland. 2010. "Bifactor models and rotations: Exploring the extent to which multidimensional data yield univocal scale scores." Journal of Personality Assessment 92(6): 544-559

Ryan, Joseph J., Christine A. Carruthers, Lori J. Miller, Gary T. Souheaver, Samuel T. Gontkovsky, and Martin D. Zehr. 2003. "Exploratory Factor Analysis of the Wechsler Abbreviated Scale of Intelligence (WASI) in Adult Standardization and Clinical Samples." Applied Neuropsychology 10(4): 252-256.

Salekin, Randall T., Craig S. Neumann, Anne-Marie R. Leistico, and Alecia A. Zalot. 2004. "Psychopathy in Youth and Intelligence: An Investigation of Cleckley’s Hypothesis." Journal of Clinical Child and Adolescent Psychology 33(4): 731-742.

Schweinle, William, William Ickes, Kathryn Rollings, and Colette Jacquot. 2010. "Maritally aggressive men: Angry, egocentric, impulsive, and/or biased." Journal of Language and Social Psychology 29(4): 399-424.

Steiger, James H. 1990. "Structural model evaluation and modification: An interval estimation approach.” Multivariate Behavioural Research 25(2): 173-180.

Tucker, Ledyard R., and Charles Lewis. 1973. "The reliability coefficient for maximum likelihood factor analysis." Psychometrika 38(1): 1-10.

Vitacco, Michael J., Craig S. Neumann, and Rebecca L. Jackson. 2005. “Testing a Four-Factor Model of Psychopathy and Its Association With Ethnicity, Gender, Intelligence, and Violence." Journal of Consulting and Clinical Psychology 73(3): 466-476. 
Vitacco, Michael J., Craig S. Neumann, and Thomas Wodushek. 2008. "The dimensions of psychopathy and intelligence: Replication with adult jail inmates." Criminal Justice and Behavior 35: 48-55.

Wechsler, David. 2008. Wechsler Adult Intelligence Scale - Fourth Edition (WASI-IV). San Antonio, TX: Pearson.

Wechsler, David. 2011. Wechsler Abbreviated Scale of Intelligence - Second Edition (WASIII). San Antonio, TX: NCS Pearson.

Wechsler, David. 1999. Wechsler Abbreviated Scale of Intelligence (WASI). San Antonio, TX: The Psychological Corporation. 


\section{Bio}

Kathryn Sharratt, MSc, ia a Senior Lecturer in Criminology at the University of Huddersfield. Her research interests and publications include emotional effects of suspicious child death cases on police investigators, assessment and rehabilitation of offenders, psychopathy and intelligence.

Daniel Boduszek, PhD is a Professor of Criminal Psychology at the University of Huddersfield. His current research interests and publications include the aspects of criminal social identity, homicidal behavior, psychopathy, prisonization, and recidivis.

Christopher Retzler, PhD is a Senior Lecturer in Psychology at the University of Huddersfield. His current research interests and publications include combining behavioural paradigms with EEG to investigate the underlying mechanisms involved in addiction. 